\title{
From Ideas and Concepts to Practice
} -Improving Effectiveness in Implementing Recommendations-

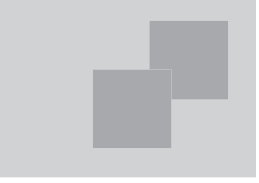

\author{
TeMS Co., Ltd., Masaharu Kitamura
}

Investigation reports on the Fukushima Accident form just one part of the numerous criticisms and recommendations that have been publicly announced with respect to reforming the way nuclear energy is harnessed. Nevertheless, a major problem has been encountered when attempts have been made to address these criticisms and put recommendations into practice. In essence, this problem is due to an absence of clearly defined measures for translating principles into practice. No matter how brilliant a recommendation may be, it will not work in practice if it is infeasible or there is no clear pathway for putting it into practice. This commentary seeks to address this problem by discussing findings related to subjects such as human factors, organizational management theory, resilience engineering, and science-technology-society (STS) studies.

\section{Introduction}

"Something important seems to be missing." This sense of discomfort persisted whenever the author heard opinions related to nuclear issues after the Fukushima Accident and read reports from the government, the private sector, and the Diet's investigation commission (NAIIC) ${ }^{1-3)}$. Certainly, it is only natural for stakeholders in the nuclear sector to be subjected to harsh criticism and urged to make fundamental changes to nuclear technologies while taking heed of recommendations. Moreover, they have a duty to address criticisms and to translate recommendations into tangible actions. However, an awareness of this was not enough to dispel the nagging sense of discomfort felt by the author.

To determine why something important seems to be missing, the author examined findings related to subjects such as human factors, organizational management theory, resilience engineering, and science-technology-society (STS) studies. This examination identified some of the causes of this sense of discomfort along with possible measures for addressing these causes. Taking the opportunity provided by the current momentum for improved nuclear safety and the ongoing development of the necessary regulatory framework, this commentary presents observations made from various perspectives that have not been explicitly taken into account to date.

DOI : $10.15669 /$ fukushimainsights.Vol.2.137

(C) 2021 Atomic Energy Society of Japan. All rights reserved.

Originally published in Journal of the Atomic Energy Society of Japan (ISSN 1882-2606), Vol. 55, No. 4, p. 212-216 (2013)

in Japanese. (Japanese version accepted: January 31, 2013) 


\section{Overview of the Author's Sense of Discomfort}

Taken as a whole, the sense of discomfort felt by the author is caused by the existence of an unclear pathway for addressing comments from investigation reports and putting recommendations into practice. In other words, it is unclear how principles should be translated into practice. The accident investigation reports only presented principles and guidelines, leaving people on the frontline to deal with the practical problems. This approach is actually the prevailing reporting style in Japan. To be fair, this approach is reasonable provided that it is meant to encourage practical on-site initiatives based on set principles and avoiding micromanagement. However, those in charge may become confused unless the principles and guidelines have proven feasible and practical measures are presented in a convincing manner. Both nuclear sector stakeholders and society as a whole may lose out in terms of time invested in exploring measures for putting recommendations into practice if they turn out to be unfeasible. The author has examined the factors behind this unclear linkage between principles and practice. The following sections examine the various factors behind the existing sense of discomfort with reference to specific examples.

\section{Rebuilding a Safety Culture}

The government's investigation committee on the accident that occurred at the Fukushima Nuclear Power Plants has strongly urged utilities, regulatory authorities, concerned bodies, council members, and other stakeholders in the nuclear sector to try to rebuild a safety culture (government, p. 429) ${ }^{a}$. The International Atomic Energy Agency (IAEA) has defined a safety culture as "the assembly of characteristics and attitudes in organizations and individuals which establishes that, as an overriding priority, protection and safety issues receive the attention warranted by their significance." Accordingly, many nuclear organizations establish basic policies, codes of conduct, and the like. Slogans and guidelines such as "safety first," "questioning attitude," and a "reporting culture" are stressed on the sites of nuclear projects. However, a certain amount of ingenuity must be exercised to translate these principles into tangible actions. In practice, it is not easy to implement measures translated from principles as the process involved requires many tradeoffs.

No recommendations have not been issued to tangibly assess the level of the safety culture (to facilitate the rebuilding process). The accident investigation report published by the government sets out specific check items (government, p. 427) for evaluating the level of the safety culture while referring to the opinion of J. Reason as a leading authority on organizational safety. However, it is still abstract and unclear how some of these items should be applied in practice. The following are a few examples of these items.

- Can management make an unwavering decision on safety?

- Is a policy in place to independently ensure safety regardless of financial standing or business performance?

- Are any imperfect conditions or risks subject to lax judgments or are they glossed over?

It is unclear what the report meant by an "unwavering" decision or to "independently ensure safety." It is also unclear what they meant by "lax" judgments in response to imperfect conditions or risks. At present, we know that the Tokyo Electric Power Company (TEPCO) misjudged the risk of major tsunamis. With the benefit of hindsight, the decision that was

\footnotetext{
${ }^{a}$ This commentary cites extensively from reports published by the government, the private sector, and the Diet's investigation commission. To save space for the references, the sources of information from these three types of reports have been simplified by recording them as "(government, p. xxx)" or "(Diet, p. yyy)," for example.
} 
taken was by no means "unwavering" and the judgment was "lax." However, the real problem concerns what can be expected in the future. Many individuals and organizations will issue warnings and share opinions on nuclear safety. Therefore, it is necessary to determine what types of responses satisfy the recommendation items as measures in order to facilitate concrete decision-making.

\section{Safety First and Litigation Risks}

Litigation risks are mentioned both in terms of the safety culture and in the context of a criticism that the regulations are captured by utilities (Diet, p. 520 and p. 525). More specifically, the risks postulated by TEPCO included measures prompted by severe accidents, shutdowns of existing reactors, and weak positions in terms of litigation (Diet, p. 525). The regulatory authorities are criticized for working in coalition with TEPCO to reduce litigation risks, which contradicts their intended mission (Diet, p. 489). Meanwhile, some have pointed out that litigation related to the safety of the Ikata Nuclear Power Plant led to a need to prepare evidence proving the safety of each nuclear power plant and that the resultant safety regulations are deprived of a mechanism for ensuring the overall safety of nuclear power plants (private sector, p. 300). In practice, this comment implies that utilities face much higher litigation risks. The author does not criticize parties who bring cases to courts. However, the recommendation that, for fear of litigation risks, a safety culture should be strongly built without a tunnel vision rings hollow. In reality, the nuclear power utilities and regulatory authorities are at a loss about what measures to be done in practice. This issue is directly related to the feasibility of the recommendations.

\section{Issue Related to the Disposal of High Level Radioactive Waste}

The issue is not explicitly mentioned in the accident investigation reports, but it is known to have an intrinsic importance to the discussion of nuclear issues. In discussions related to nuclear energy, the author and his colleagues have often seen the lack of support for nuclear power justified by claims that the issue of disposal remains unresolved (or indecisiveness concerning nuclear power caused by confusion over the issue). In other words, the resolution of the disposal issue is a prerequisite for formulating a nuclear policy. Meanwhile, the Atomic Energy Commission of Japan asked the Science Council of Japan to discuss this issue. The council responded ${ }^{4)}$ by saying that it is vital to present an overriding principle for the nuclear policy to gain broad public understanding. Here, the resolution of the disposal issue is premised on formulating the nuclear policy. These two issues form a circular relationship in which a failure to resolve one issue makes it impossible to address the other. Finding a solution to such a relationship is extremely difficult. The council went on to recommend that a venue be established to allow an epistemic community of autonomous scientists to engage in specialized discussions and that such discussions be coordinated by an impartial third party. These recommendations seem reasonable in principle, but it is unclear how they should be put into practice.

\section{Lessons Learned from the TMI Accident}

More than one investigation report discusses the Fukushima Accident in relation to the Three Mile Island (TMI) Accident. A report by the NAIIC devoted many pages to explaining the reform of US regulatory bodies after the TMI Accident. It points out that "the Japanese 
regulatory system lagged behind that of the United States and France after the reform ... which led to the failure to prevent the Fukushima Accident and its escalation" (Diet, p. 573). This is a valid comment, but it should be noted that considerable technical measures were implemented in Japan following the TMI Accident. Without going into too much detail, it is safe to say that Japan was relatively quick to introduce the safety parameter display systems (SPDSs) and alarm filters required to classify alerts by their degree of importance. Looked at differently, recommendations concerning hard measures can be easily applied in Japan because of clarity in terms of the way they should be implemented. In contrast, recommendations regarding regulations, safety management, and other soft measures arguably tend to be applied more slowly due to a lack of clarity in terms of the way they should be implemented. Recommendations that rely on principles may have been delayed for this reason.

A careful look at the report ${ }^{5)}$ published by the President's Commission on the accident at Three Mile Island demonstrates that measures comparable to the cultivation of safety culture, which would finally attract global attention only after the Chernobyl Accident, had already been discussed extensively.

The following extracts are particularly relevant to safety culture.

- The TMI Accident could most likely have been prevented had there been a sincere response to the numerous warnings that had already been issued. (p. 29)

- The agency should be directed to employ a broader definition of matters relating to safety that considers thoroughly the full range of safety matters. (p. 63)

- The Commission recognizes that merely meeting the requirements of a government regulation does not guarantee safety. (p. 68)

This means that the nuclear industry in Japan and abroad had already been advised of a concept tantamount to safety culture and that there was no reason why the world had to wait until the shock caused by the Chernobyl Accident before action was taken. This fact clearly highlights the importance of translating principles into practice.

\section{Narrowing Down the Factors Involved in the Case Studies}

The case studies presented in the previous chapter demonstrate the difficulty involved in making criticisms and recommendations feasible and providing substance to them. The same problem can be commonly observed with respect to many other criticisms and recommendations. Some factors are unique to certain issues, but case studies can usually be narrowed down to a relatively few common factors.

\section{Common Factors}

One of these common factors is the difficulty involved in implementing safety measures. This challenge is directly associated with not only safety culture, but also litigation risks and the lessons learned from the TMI Accident. Needless to say, safety assurance must be considered in terms of both the prevention of accidents and their escalation in normal operation and the performance of an emergency response to any accidents. One other type of issue is that the disposal of high level radioactive waste also presents a core challenge in relation to the difficulty involved in ensuring the long-term safety of radioactive waste buried underground. In this respect, many criticisms and recommendations are related to the technical challenges associated with ensuring safety. 
In today's society, it is no longer possible for nuclear experts to make a decision on their own as to whether nuclear solutions can be introduced in society even if engineers have determined that such solutions are safe. The conventional approach in which a group of experts explain their decisions to regulatory bodies, municipalities, local residents, and the general public is no longer a viable means of gaining widespread public support for the level of safety that has been achieved. The inevitable advent of a "republic of trans-science" (6) that involves a diverse group of non-experts has already been clearly pointed out with respect to resolving the friction between technologies and society. Investigation reports and the like on the Fukushima Accident reflect these findings to some extent. However, the author has not yet observed any discussions that delve deeper and recommend how a diverse group of nonexperts can be involved in practical measures to address this issue.

Moreover, issues related to litigation risks and the disposal of high level radioactive waste must be considered with respect to environmental ethics, intergenerational ethics, administrative adequacy, and many other issues, as well as safety issue. Naturally, these issues must also be resolved in collaboration with not only nuclear experts, but also a diverse group of experts from other fields and various stakeholders. Given this, implementation of safety and collaborating with people who are not themselves nuclear specialists emerge as two important common factors. These factors are discussed in greater detail below.

\section{Factors Concerning Safety}

First, we need to recognize that too many discussions and proposals are made without actually providing a clear definition of safety. As has already been mentioned, safety is a concept that should not be defined in a manipulative manner and cannot be defined based on evidence $^{7)}$. This extremely important implication must be understood. Defining safety as being equivalent to the absence of unacceptable risks is nothing but a paraphrase that is essentially difficult to prove. Therefore, discussions on safety must be conducted by clarifying an operative definition of safety (or by clarifying measures for implementing safety). Unfortunately, the concept of safety is not defined when people discuss safety culture or engage in disputes over accountability for safety in litigations. This leads to confusion in relation to conflicting perspectives on safety. Future discussions should at least be based on a clearer definition of safety. The seemingly obvious basic concept of safety is evolving at the frontline of research on safety. Measures to implement safety are transforming considerably to keep pace with this evolution. To avoid any digression, the author leaves detailed explanations of these trends to the reference materials ${ }^{8,9)}$. However, necessary explanations will be provided later in relation to the commentary. References to new findings in relation to safety may help us to move closer to a shared perspective and ease conflict arising from firmly held opinions.

\section{Factors Concerning the Collaboration with Non-Nuclear Experts}

Even if investigations focus on the causes of the Fukushima Accident and ways to prevent a recurrence, a vast range of issues must be addressed. Such issues include organizational management, desirable regulations, and the involvement of local municipalities and citizens. A great deal of knowledge has been accumulated in terms of the theory and practice of various disciplines, including organizational management, social relations management, the perception and communication of nuclear risks, and regulatory science. Realistically, it is difficult for utilities and regulatory bodies to undergo training from specialists in their respective disciplines before applying the accumulated theoretical and practical knowledge in practice 
(as they should). Utilities and regulatory bodies usually end up facing practical issues after skimming through introductory guides that happen to be available or participating in training sessions for beginners at most.

There is considerable demand for measures to resolve issues related to the relationship between nuclear energy and society in broad terms, including the dismantling of the collusive "nuclear village," the provision of transparent explanations to society, and the promotion of greater citizen participation. Nevertheless, it is difficult for nuclear experts to independently define the extent to which certain measures would satisfactorily address recommendations and how the difficulties involved in implementing these measures should be overcome. Putting such measures into practice is another problem. It is only natural for experts in the humanities and social sciences ${ }^{6}$ to be encouraged to play active roles in the resolution of problems related to the relationship between nuclear energy and society.

Any such solutions would require us to build a platform for interdisciplinary collaboration and take measures to ensure its effectiveness. The necessary practice of intercultural communication ${ }^{10)}$ is itself a difficult task. Consequently, issues related to nuclear power are too difficult for nuclear experts to address on their own, so collaboration with experts in the humanities and social sciences is necessary. The additional difficulty involved in achieving such collaboration compounds the challenge. Stakeholders in the nuclear sector must be prepared to deal with this twofold challenge.

\section{Measures Implemented in Response to Factors}

\section{Redefining Safety}

As mentioned above, discussions concerning safety are intrinsically difficult. Measures to address or mitigate this difficulty have been explored with reference to the progress made in recent research on safety.

\section{(1) Transition from Safety-I to Safety-II}

Safety-I is based on the conventional view of safety. Widely known definitions include a "peaceful condition without any hazards" (Kojien (Japanese dictionary)) and the "absence of unacceptable risks" (IAEA, etc.). However, such definitions all assume a static state without any undesirable conditions. In contrast, Safety-II is considered important among researchers who advocate the concept of safety with an emphasis on resilience. It is defined as the "continuation of system operations while avoiding any catastrophic conditions ${ }^{8)}$." In other words, Safety-II represents a dynamic and proactive concept that takes into consideration the necessary responses to disturbances, failures, and other such problems. Under the definition of Safety-II, accident prevention and damage minimization are the most important and necessary conditions for continuing operations. However, Safety-II does not set static safety as the ultimate goal, so it avoids ideological obsession. Instead, it considers the conditions that are desirable both during normal operations and in the event of accident. This definition is also consistent with the idea of defense in depth. The concept of Safety-II will help to ease tensions arising from conflicting ideas considerably in the pursuit of safety.

(2) Ideas behind defense in depth and Safety-II

Defense in depth is widely known to be based on the assumption that the preceding level of protection fails. The IAEA defines the five levels of defense in depth as follows: (1) prevention of abnormal operation and failures; (2) control of abnormal operation and detection of 
failures; (3) control of accidents within the design basis; (4) control of severe plant conditions, including prevention of accident progression and mitigation of the consequences of severe accidents; and (5) mitigation of radiological consequences of significant releases of radioactive materials. Some argue that a higher level of protection is not necessary if each level can perform its role perfectly. Certainly, it is desirable for each level to be able to ensure sufficient safety by itself. However, based on the primary idea of defense in depth, it is sensible to prepare higher levels of protection because no mechanical system or human operator is perfect.

Levels 4 and 5 are taken into consideration based on the assumption that the levels of defense stipulated in Levels 1 through 3 could be breached, even if the degree of probability is low. Before the Fukushima Accident, it was popularly reasoned that an event based on many extreme assumptions need not be considered (as the event would never take place), but that line of reasoning has been discredited. Instead, a more realistic and logical approach based on Safety-II would be as follows. Most failures and disturbances can be dealt with by Levels 1 through 3. Beyond that, Level 4 can protect local residents from any events that may overwhelm the levels of defense provided up to Level 3. Furthermore, even if the protection afforded by Level 4 fails due to a rare event, the disaster management plan in place will secure sufficient time for the evacuation of residents while also avoiding the type of chaos experienced during the Fukushima accident.

Nuclear safety experts have known about the concept of defense in depth since long before Safety-II gained recognition as an important philosophy. However, this concept could not be explained well due to inconsistencies in the understanding of safety. One investigation report explains in detail how it complicated the explanation of acceptable risk levels and severe accidents (government, p. 311 and p. 321). Safety-II will serve as a mediating concept that allows nuclear experts and citizens to gain a shared understanding of defense in depth while avoiding any discrepancies. Unfortunately, public trust in nuclear experts has already been damaged, so it is not viable for these experts alone to share the same understanding of the concept with the public. A more desirable approach would be to ask non-nuclear experts who specialize in safety to serve as intermediaries.

An important suggestion should be noted in relation to the argument that "appropriate safety and disaster control measures must be prepared for accidents and disasters that result in extensive damage regardless of their probabilities" (government, p. 413). Preparing artificial structures against any events "regardless of their probabilities" seems an almost impossible requirement. However, one must give careful thought to the interpretation of the modifying word "appropriate." It should also be noted that this requirement is only possible if Safety-II is adopted as the basic guideline.

\section{Suggestions in Relation to Interdisciplinary Collaboration}

Citizen participation is being explored on many fronts in relation to political decision-making involving nuclear technologies. National debate has already been attempted thanks to the efforts made by experts in the humanities and social sciences. Nevertheless, many issues remain to be resolved in terms of involving citizens in decision-making, which would certainly be a desirable development. In addition to directly importing methods that have been cultivated abroad, we should also explore measures that fit the reality in Japan. We are keenly aware that the Fukushima Accident stems in part from the blind introduction of nuclear power facilities developed in the United States. Despite the intention to strengthen citizen participation, the same mistake might be repeated even with proven overseas methods if they are adopted in Japan as is. 
As the NAIIC chair, Mr. Kurokawa drew a considerable response when he commented in an English report that the Fukushima Accident stemmed from a unique aspect of Japanese culture. We obviously need to be careful not to ascribe technical problems to cultural uniqueness. Nevertheless, cultural considerations are necessary to translate codified principles and guidelines into practice and to form a shared understanding with the public.

\section{Conclusions}

Simply put, all of the problems discussed in this commentary are associated with a poor interface between principles and the reality of the situation. In his capacity as the chair of the investigation committee, Mr. Hatamura rightly pointed out that the format alone does not ensure proper functioning and that the purpose cannot be shared by simply building a system (government, p. 446). The nuclear community must recognize its responsibility to convert this comment into reality. They must determine which principles can be effectively put into practice and then recommend them both on the frontline and to the relevant bodies. However, fulfilling this responsibility would be difficult for engineers and technicians alone. Consequently, there are high hopes that expertise from a wide spectrum of disciplines, including the humanities and social sciences, will also play a part. However, the first move must be made by stakeholders in the nuclear sector with due consideration given to their responsibilities.

\section{References}

1) Investigation Committee on the Accident at the Fukushima Nuclear Power Stations: Final Report (2012) [in Japanese], http://icanps.go.jp/post-2.html (accessed January 30, 2013).

2) Rebuild Japan Initiative Foundation: Investigation Report by the Independent Investigation Commission on the Fukushima Daiichi Nuclear Accident [in Japanese], Discover, (2012).

3) Report by the National Diet of Japan Fukushima Nuclear Accident Independent Investigation Commission [in Japanese], (2012), http://warp.da.ndl.go.jp/info:ndljp/pid/3856371/naiic.go.jp/index.html (accessed January 30, 2013).

4) Science Council of Japan: Response Regarding the Disposal of Highly Radioactive Waste [in Japanese], (2012).

5) J. G. Kemeny: Report of the President's Commission on the Accident at Three Mile Island (1979).

6) T. Kobayashi: Age of Trans-Science [in Japanese], NTT Publishing (2007).

7) T. Kinoshita: Risk Analysis of the Fukushima Nuclear Accident [in Japanese], Journal of the Atomic Energy Society of Japan, 53 (7), 465-472 (2011).

8) M. Kitamura (translation supervisor): Resilience Engineering - Concepts and Precepts, Japanese Union of Scientists and Engineers (2012).

9) M. Kitamura: Re-engineering of Nuclear Safety Logic and Resilience-based Studies of Safety [in Japanese], Journal of the Atomic Energy Society of Japan, 54 (11), 721-726 (2011).

10) E. T. Hall: Beyond Culture, Anchor Books (1977). 